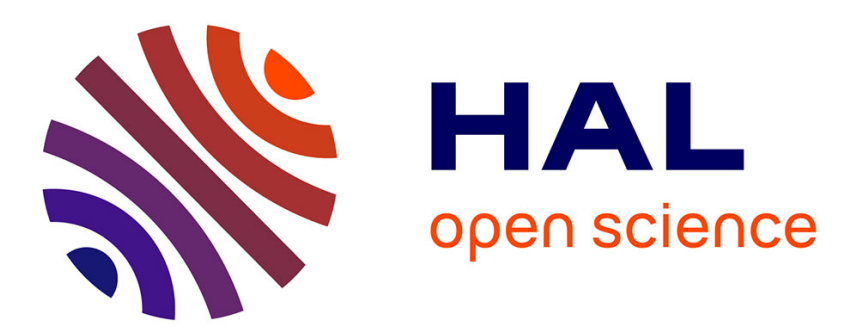

\title{
Transition from multiplicity to singularity of steady natural convection in a tilted cubical enclosure
}

Juan Felipe Torres, Daniel Henry, Atsuki Komiya, Shigenao Maruyama

\section{To cite this version:}

Juan Felipe Torres, Daniel Henry, Atsuki Komiya, Shigenao Maruyama. Transition from multiplicity to singularity of steady natural convection in a tilted cubical enclosure. Physical Review E: Statistical, Nonlinear, and Soft Matter Physics, 2015, 92, pp.023031. 10.1103/PhysRevE.92.023031. hal-01298322

\section{HAL Id: hal-01298322 \\ https://hal.science/hal-01298322}

Submitted on 11 Apr 2016

HAL is a multi-disciplinary open access archive for the deposit and dissemination of scientific research documents, whether they are published or not. The documents may come from teaching and research institutions in France or abroad, or from public or private research centers.
L'archive ouverte pluridisciplinaire HAL, est destinée au dépôt et à la diffusion de documents scientifiques de niveau recherche, publiés ou non, émanant des établissements d'enseignement et de recherche français ou étrangers, des laboratoires publics ou privés. 


\title{
Transition from multiplicity to singularity of steady natural convection in a tilted cubical enclosure
}

\author{
Juan F. Torres* \\ Mechanical Systems Laboratory, Corporate Research \& Development Center, Toshiba Corporation, 1, Komukai-Toshiba-cho, \\ Saiwai-ku, Kawasaki 212-8582, Japan \\ Daniel Henry \\ Laboratoire de Mécanique des Fluides et d'Acoustique, CNRS/Université de Lyon, École Centrale de Lyon/Université Lyon 1/INSA de Lyon, \\ ECL, 36 avenue Guy de Collongue, 69134 Ecully Cedex, France
}

Atsuki Komiya and Shigenao Maruyama

Institute of Fluid Science, Tohoku University, 2-1-1, Katahira, Aoba-ku, Sendai, Miyagi 980-8577, Japan

(Received 8 May 2015; published 28 August 2015)

\begin{abstract}
The transition from the complex Rayleigh-Bénard convection to the simple heated-from-the-sides configuration in a cubical cavity filled with a Newtonian fluid is numerically studied. The cavity is tilted by an angle $\theta$ around its lower horizontal edge and is heated and cooled from two opposite tilted sides. We first analyze the effect of a marginal inclination angle on quasi-Rayleigh-Bénard convection $\left(\theta \approx 0^{\circ}\right)$, which is a realistic physical approximation to the ideal Rayleigh-Bénard convection. We then yield the critical angles where multiple solutions that were initially found for $\theta \approx 0^{\circ}$ disappear, eventually resulting in the single steady roll solution found in the heated-from-the-sides configuration $\left(\theta=90^{\circ}\right)$. We confirm the existence of critical angles during the transition $\theta: 0^{\circ} \rightarrow 90^{\circ}$, and we demonstrate that such angles are a consequence of either singularities or collisions of bifurcation points in the Rayleigh-number- $\theta$ parameter space. We finally derive the most important critical angles corresponding to any Newtonian fluid of Prandtl number greater than that of air.
\end{abstract}

DOI: 10.1103/PhysRevE.92.023031

\section{INTRODUCTION}

The phenomenon of buoyancy-driven natural convection in closed environments is ubiquitous. In nature, for example, this phenomenon can be found in large scales in the Earth's mantle [1] and the atmosphere of planets [2]. In engineering, for example, natural convection in enclosures can be used in microfluidic DNA analysis devices $[3,4]$ and in temperaturecontrol strategies for solar-energy collectors [5] and nuclear reactors [6]. Therefore, there exists a great diversity of buoyancy-driven flows in enclosures that are of great interest in science and technology [7,8]. Rayleigh-Bénard convection (RBC) is arguably the most studied form of natural convection in cavities [9-12] since the pioneering work of Bénard and Rayleigh a century ago $[13,14]$. RBC results from the development of convective instabilities due to a nonuniform temperature distribution in a plane horizontal fluid layer heated from below. This phenomenon is complex due to the diversity of steady flows that can arise for given geometries and heating configurations $[15,16]$. On the other hand, steady natural convection in a cavity heated from the sides, or what we call in this paper heated-from-the-sides convection (HSC), is a much simpler problem that accounts for a single convective roll [17]. In spite of the great number of published work on both subjects, the transition from the complex RBC to the simple HSC, e.g., as that shown in Fig. 1, is still a fundamental physics problem whose mechanism is not fully understood [18-24]. In an attempt to explain this transition, we previously conducted a preliminary, but detailed, bifurcation analysis for water (Prandtl number $\mathrm{Pr}=5.9$ ) in a cubical enclosure and reported several critical angles where stable solutions cease

\footnotetext{
*torres.jf@outlook.com, felipe.torres@ toshiba.co.jp
}

PACS number(s): 44.25.+f, 47.20.Bp, 47.20.Ky

to exist [25]. It is well-known, however, that the dynamics of RBC in an enclosure is greatly dependent on the Prandtl number, i.e., the ratio of momentum diffusivity to thermal diffusivity expressed as $\operatorname{Pr}=v / \alpha$, where $v$ and $\alpha$ are the kinematic viscosity and thermal diffusivity, respectively.

In this paper, we provide new insight into the mechanism behind the transition from RBC to HSC for any Newtonian fluid with a Prandtl number larger than that of air. We first give a detailed description of RBC and HSC for air $(\operatorname{Pr}=0.71)$, since it is a fluid of great relevance to human beings. We then discuss the rich dynamics found during the RBC-HSC transition. The main objective of this study is to reveal the mechanism behind this transition for steady flow. We finally derive the main critical angles as a function of the Prandtl number for $0.71 \leqslant \operatorname{Pr} \rightarrow \infty$.

\section{PROBLEM DESCRIPTION}

In this study, we keep the same cubical configuration, system of coordinates, and notation as described in Ref. [25]. For RBC in noncubical cavities (aspect ratio $A \neq 1$ ) an uneven shear stress exerted from the sidewalls favors certain groups of solutions [26]. In contrast, $\mathrm{RBC}$ in a cubical enclosure ( $A=1$ ) yields symmetrical solutions that are only influenced by the Rayleigh number, Prandtl number, and the boundary conditions [12]. Thus, due to its rich symmetrical properties $[15,16,27]$, the cubical cavity is a suitable geometry to study the RBC-HSC transition [28].

We consider a tilted cubical enclosure completely filled with a Newtonian fluid, which is heated and cooled from two opposite isothermal sides of the cavity. The Newtonian fluid is taken as a pure fluid in order to avoid undesired instabilities due to the Soret effect [29-31]. The cavity forms 


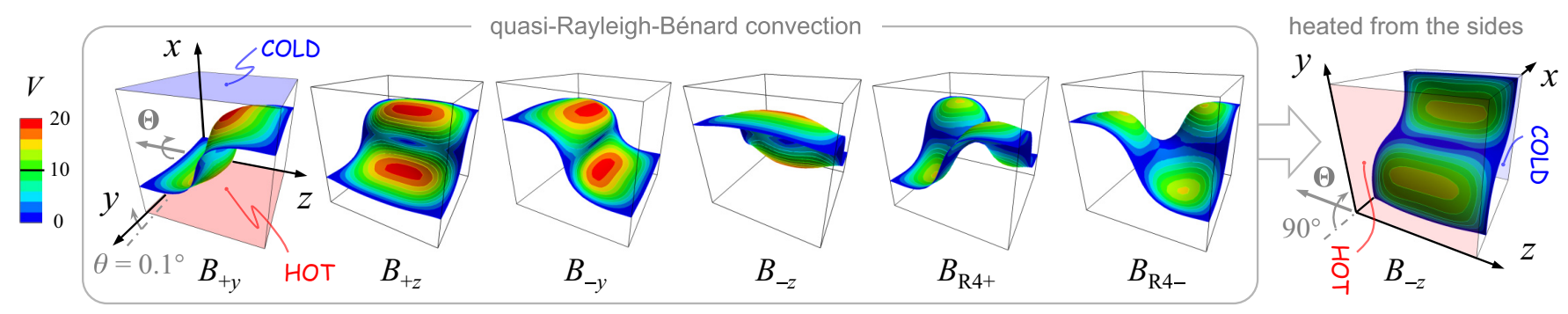

FIG. 1. (Color online) Stable solutions found in a cubical cavity filled with air at $\mathrm{Ra}=10000$ in quasi-Rayleigh-Bénard convection $\left(\theta=0.1^{\circ}\right)$ and heated-from-the-sides configuration. Multiple solutions are found in the former case, whereas a single solution is found in the latter case. The magnitude of the dimensionless velocity is plotted on the mean-isothermal surface $T=\left(T_{H}+T_{C}\right) / 2$.

an angle $\theta$ between the horizontal plane and its isothermal sides, as shown in Fig. 1 for the inclination angles $\theta=0.1^{\circ}$ $(\sim 1.7 \mathrm{mrad})$ and $\theta=90^{\circ}$. The inclination vector can then be written in vectorial form as $\boldsymbol{\Theta}=-\theta \boldsymbol{e}_{z}$, where $\boldsymbol{e}_{z}$ is a unit vector in the $z$ direction. The Cartesian system of coordinates is fixed to three orthogonal edges of the cavity, as shown in Fig. 1, with its $z$ axis overlapping with a lower horizontal edge. The sides $y z$ are kept isothermal at temperatures $T_{H}$ and $T_{C}$ $\left(T_{H}>T_{C}\right)$, while the remaining sidewalls $x z$ and $x y$ are kept adiabatic to avoid heat loss through these boundaries. This heating configuration generates a buoyancy-driven convective flow inside the cavity. Our goal is to clarify the behavior of the stable flow solutions found in the cubical cavity during the transition from $\mathrm{RBC}$ to $\operatorname{HSC}\left(\theta: 0^{\circ} \rightarrow 90^{\circ}\right)$.

\section{NUMERICAL TECHNIQUES}

We have recently developed a continuation procedure to study steady natural convection in a parallelepiped enclosure [26]. The continuation method is based on a Jacobianfree Newton solver [32]. We solve the governing equations (conservation of mass, momentum, and energy) with a three-dimensional spectral finite element method under the Boussinesq approximation $[33,34]$ while taking into account the acceleration of gravity, which has components along two edges of the cubical cavity. Our continuation method yields comprehensive bifurcation diagrams, which describe the behavior of the flow instability for a wide range of Rayleigh numbers. Remember that the ratio of the buoyancy driving force to its dissipative factors (diffusion of temperature and momentum) is described by the Rayleigh number $\mathrm{Ra}$ globally defined as $\mathrm{Ra}=g \beta\left(T_{H}-T_{C}\right) L^{3} /(v \alpha)$, where $g$, $\beta, T$, and $L$ are the acceleration of gravity, the thermal expansion coefficient, the temperature, and the edge length of the cubical cavity. The bifurcation diagrams, in contrast to three-dimensional flow solutions for a given Ra (see Fig. 1), describe the behavior of the stable solutions in the Ra-parameter space.

\section{RESULTS}

Quasi-Rayleigh-Bénard convection. In spite of the claim by most experimentalists and theoreticians that their subject of study is RBC, the configuration where the heated surface is perfectly horizontal is an ideal condition impossible to achieve. Even in a controlled experimental setup where the cavity is intended to be horizontal [15], a marginal inclination is always present. This marginal inclination is small but physically significant [35,36], and it is a determining factor for the stable solutions found when the Rayleigh number $\mathrm{Ra}$ is gradually increased [19,37-40]. Therefore, we shift our attention from the ideal RBC to the more realistic quasi-Rayleigh-Bénard convection (QRBC). Figure 1 shows the multiple solutions that arise in $\mathrm{QRBC}\left(\theta=0.1^{\circ}\right)$ in a cavity filled with air $(\operatorname{Pr}=0.71)$; the magnitude of the dimensionless velocity vector is plotted as a contour on the mean-isothermal surface. As shown in this figure, six stable solutions exist in QRBC $\left(\theta=0.1^{\circ}\right)$ for $\mathrm{Ra}=10000$ : four stable transverse-roll solutions $\left(B_{+y}, B_{+z}\right.$, $\left.B_{-y}, B_{-z}\right)$ and two four-roll solutions ( $B_{\mathrm{R} 4+}$ and $\left.B_{\mathrm{R} 4-}\right)$.

Figure 2 shows the bifurcation diagrams in the range $\mathrm{Ra} \leqslant$ 100000 for RBC and QRBC. The transition between these two natural convective states, i.e., $\theta: 0^{\circ} \rightarrow 0.1^{\circ}$, is clearly depicted in this figure. See Ref. [16] for a thorough description of the RBC configuration. In Fig. 2, the horizontal velocity $w$ at the reference point $\left(x_{1}, y_{1}, z_{1}\right)=(0.90,0.79,0.90)$ is used as representative parameter to describe the stable flow solutions as a function of Ra. The solid lines and dashed or dotted lines represent stable and unstable steady-flow solutions, respectively. The multiplicity of the problem is clearly depicted by numerous stable branches. In Fig. 2(a), the dynamics of the system is shown for $2000 \leqslant \mathrm{Ra} \leqslant 6000$, focused on the solutions around the primary instability threshold $P_{1}$. In Fig. 2(b), the results are expanded to $\mathrm{Ra} \leqslant 100000$ focusing on the stable solutions (unstable segments after a stabilizing pitchfork bifurcation are also included). In insets (c), (d), and (e), the transition from RBC to QRBC is shown in the vicinity of some bifurcation points, where it is more pronounced.

It is interesting to see from Fig. 2 that the slight inclination in QRBC breaks the degeneracy of the stable solutions found in RBC as follows. The transverse stable rolls $B_{-z}$, whose rotation vector is in the same direction as the inclination vector $\boldsymbol{\Theta}$ (see Fig. 1), form a leading branch starting from $\mathrm{Ra} \rightarrow 0$. The branch $B_{+z}$ consisting of transverse rolls, whose rotation vector is opposite to $\boldsymbol{\Theta}$, develops from the saddle-node bifurcation $C_{+z}$, and is stabilized at the pitchfork bifurcation $S_{+z}$, as shown in Fig. 2(c). The semitransverse stable rolls $B_{ \pm y}$, whose rotation axis is perpendicular to $\Theta$ for $\theta=0^{\circ}$, develop from the saddle-node bifurcations $C_{ \pm y-z}$. The branches of four-roll solutions $B_{\mathrm{R} 4 \pm}$, which for $\theta=0^{\circ}$ start unstable at $S_{\mathrm{R} 4}$ [Fig. 2(a)] but are stabilized at other pitchfork bifurcations [outside the range of $\mathrm{Ra}$ in Fig. 2(a)], now evolve as stable branches for $\theta=0.1^{\circ}$ from the saddle-node bifurcations $C_{\mathrm{R} 4 \pm}$. The transition from a pitchfork in RBC to a saddle-node bifurcation in $\mathrm{QRBC}$ is then observed for $B_{+z}, B_{ \pm y}$, and 

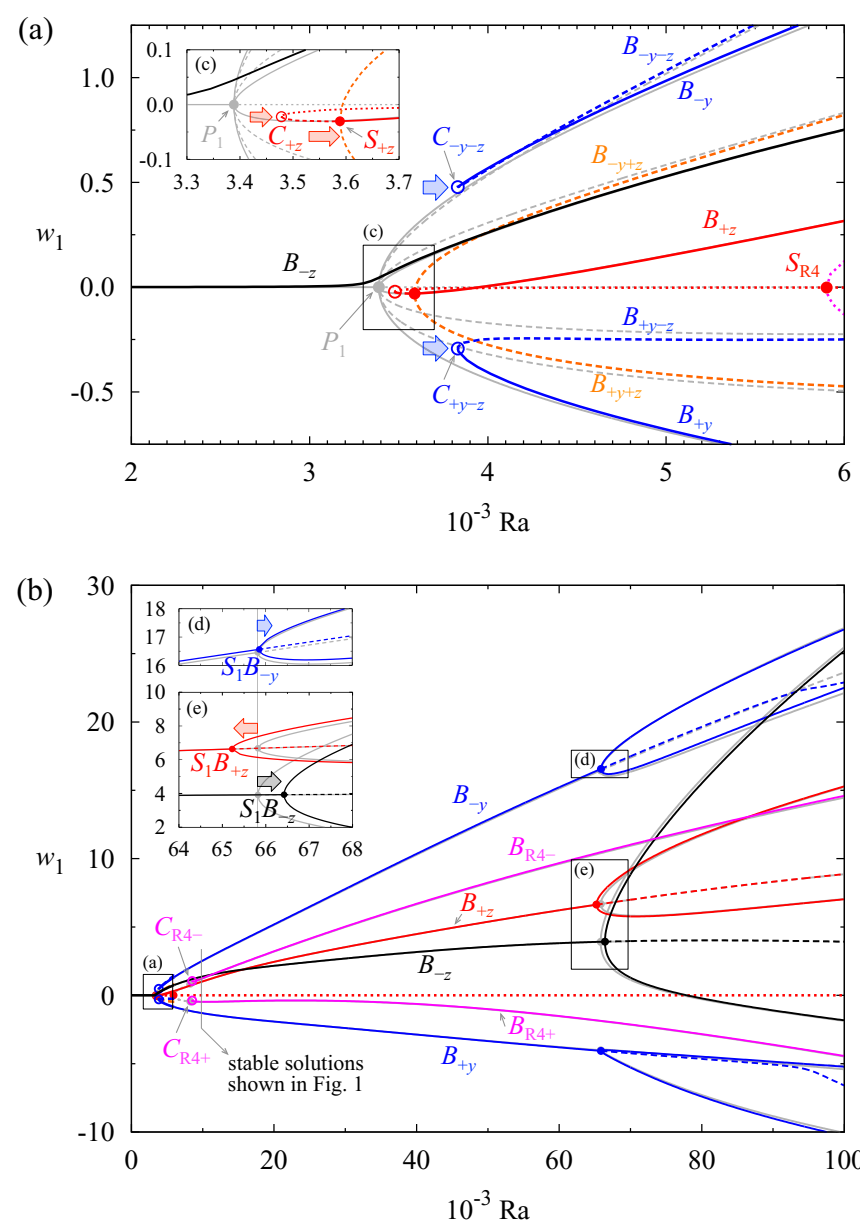

FIG. 2. (Color online) Bifurcation diagrams showing the multiple stable solutions found in $\operatorname{RBC}\left(\theta=0^{\circ}\right.$, gray lines starting at $\left.P_{1}\right)$ and $\mathrm{QRBC}\left(\theta=0.1^{\circ}\right.$, color online) in a cubical cavity filled with air. The velocity $w$ at a reference point is plotted as a function of $\mathrm{Ra}$ for $2000 \leqslant \mathrm{Ra} \leqslant 6000$ (a) and $\mathrm{Ra} \leqslant 100000$ (b). The insets (c), (d), and (e) show the transition between RBC and QRBC around the main bifurcations. The thick arrows indicate the translation of the critical thresholds as $\theta$ is increased.

$B_{\mathrm{R} 4 \pm}$. Moreover, in Figs. 2(d) and 2(e) we see that the slight inclination induces a translation in the Ra-parameter space of the secondary bifurcations $S_{1} B_{+z}, S_{1} B_{-z}$, and $S_{1} B_{-y}\left(S_{1} B_{+y}\right)$. These translations, as well as those translations around $P_{1}$, are indicated by thick arrows in Fig. 2.

Heated-from-the-sides convection. Figure 2 illustrates the multiplicity that characterizes QRBC, i.e., numerous stable solutions exist for a given Ra. This multiplicity is, however, bound to disappear when the cavity is tilted by $90^{\circ}$, as shown in Fig. 1 by the only stable solution found for HSC. The convective regime of HSC can then be represented by a single unbifurcated branch, as shown in Fig. 3(a). The light gray branches in this figure correspond to RBC and are included to add a contrast between the complexity of RBC and simplicity of HSC.

$R B C-H S C$ transition for air. The transition from RBC to HSC, i.e., $\theta: 0^{\circ} \rightarrow 90^{\circ}$, can be described by tracing the thresholds $\mathrm{Ra}_{c}$ of the bifurcation points as $\theta$ is increased. The resulting loci, or trajectories, are shown in Fig. 3(b)
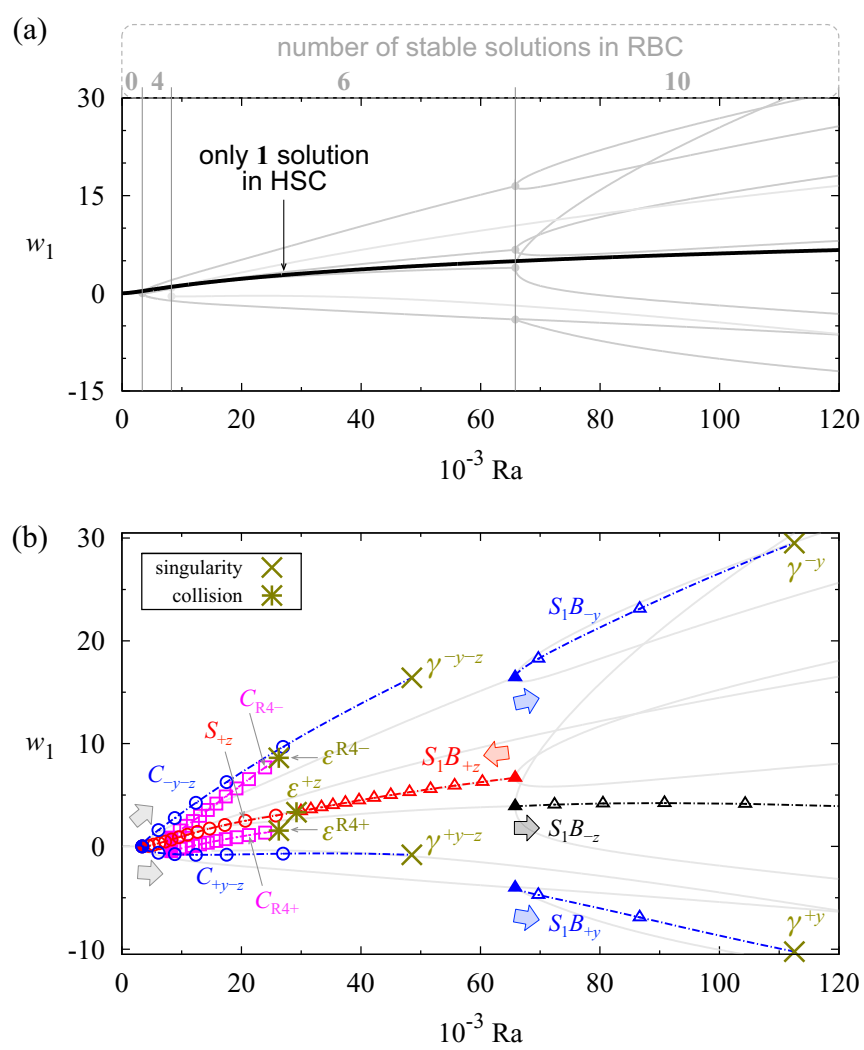

FIG. 3. (Color online) Transition from RBC to HSC for a cavity filled with air. (a) The single branch found in HSC $\left(\theta=90^{\circ}\right)$ is depicted by a single solid black line, while the multiple branches found in RBC are depicted by light-gray lines. (b) The loci of the most relevant bifurcations are shown every $\Delta \theta=1^{\circ}$ (open points) starting from $\theta=0^{\circ}$ (solid points). The circles indicate those bifurcations that evolve from the primary instability threshold $P_{1}$ at $\theta=0^{\circ}$; the squares indicate the saddle-node points where the stable four-roll solutions evolve from; the triangles indicate those solutions related to the bifurcation point $S_{1} B_{ \pm y}$ and $S_{1} B_{ \pm z}$. The collisions $\epsilon$ and singularities $\gamma\left(d \mathrm{Ra}_{c} / d \theta \rightarrow \infty\right)$ are plotted using $*$ and $\times$, respectively.

by line points; the points show the bifurcations for the tilt increase $\Delta \theta=1^{\circ}$. The results shown in Fig. 3(b) are of great interest because they demonstrate that the mechanism behind the RBC-HSC transition involves collisions $\epsilon$ and singularities $\gamma$ of different bifurcation points as the inclination of the cavity is increased, eventually resulting in the single stable solution branch shown in Fig. 3(a).

The dynamics of these bifurcations as the inclination of the cavity gets larger is described as follows. For the $B_{+z}$ rolls unfavored by $\Theta$, the stability threshold $S_{+z}$ increases while $S_{1} B_{+z}$ decreases, resulting in the collision $\epsilon^{+z}$ at $\theta_{c}^{+z} \approx 12.47^{\circ}$ [shown in Fig. 3(b) using * ]. We verified that, in fact, this collision annihilates the stable solutions for this group of solutions. Meanwhile, for the $B_{ \pm y}$ rolls, which are perpendicular to $\Theta$ when $\theta=0^{\circ}$, both $C_{ \pm y-z}$ and $S_{1} B_{ \pm y}$ shift to a larger stability threshold, but the latter at a higher rate than the former. This leads to the singularities $d \mathrm{Ra}_{c} / d \theta \rightarrow \infty$ at $\theta_{c}^{ \pm y-z} \approx 5.56^{\circ}$ for $C_{ \pm y-z}$ and $\theta_{c}^{ \pm y} \approx 2.44^{\circ}$ for $S_{1} B_{ \pm y}$. These singularities are, respectively, denoted as $\gamma^{ \pm y-z}$ and $\gamma^{ \pm y}$ [shown in Fig. 3(b) using $\times$ ]. Concerning the stable 


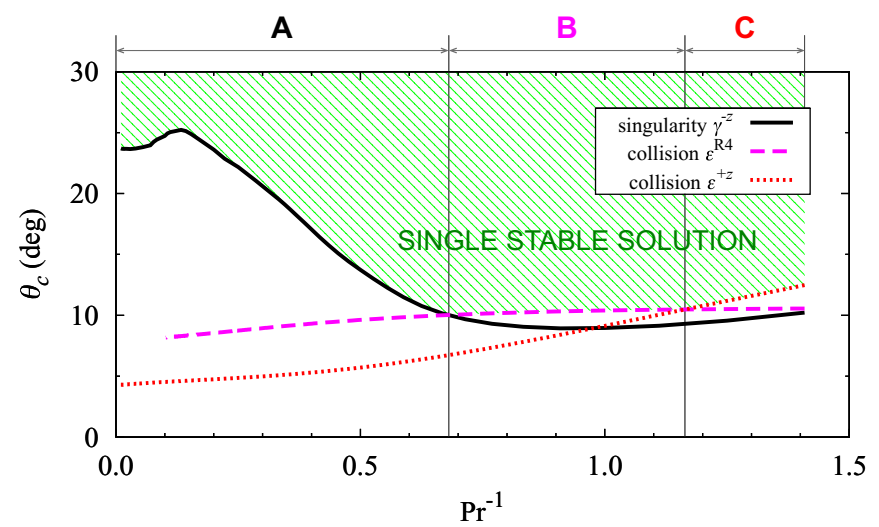

FIG. 4. (Color online) Collisions $\epsilon^{+z}, \epsilon^{\mathrm{R} 4}$, and singularity $\gamma^{-z}$ for any Newtonian fluid of Pr greater than that of air, i.e., $0.71 \leqslant \operatorname{Pr} \rightarrow$ $\infty$. The inverse of Prandtl number $\operatorname{Pr}^{-1}$ is given in the abscissa. The transition from multiple solutions found in $\mathrm{RBC}$ to a single solution found in HSC corresponds to the largest critical angle $\theta_{c}$ at a given value of Pr.

solutions on the branches $B_{-z}$, the locus of $S_{1} B_{-z}$ has the singularity $\gamma^{-z}$ at $\theta_{c}^{-z} \approx 10.22^{\circ}$ [outside the range of Ra in Fig. 3(b)]. Furthermore, concerning the stable four-roll solutions on the branches $B_{\mathrm{R} 4 \pm}$, the saddle-node bifurcations $C_{\mathrm{R} 4 \pm}$ increase with the inclination until they rendezvous with Hopf bifurcations [whose loci are omitted in Fig. 3(b)], resulting in the collisions $\epsilon^{\mathrm{R} 4 \pm}$ (written as $\epsilon^{\mathrm{R} 4}$ in the following) that annihilate the stable four-roll solutions at $\theta_{c}^{ \pm \mathrm{R} 4} \approx 10.56^{\circ}$.

Therefore, the largest critical angle, which for air corresponds to the collision critical angle $\theta_{c}^{+z} \approx 12.47^{\circ}$, delimits the final transition from a multiple-stable-solution to a singlestable-solution configuration (characteristic of RBC and HSC, respectively).

RBC-HSC transition for a fluid of $0.71 \leqslant \operatorname{Pr} \rightarrow \infty$. In order to obtain a more comprehensive understanding of the transition between RBC and HSC, we investigate the collisions $\epsilon^{+z}, \epsilon^{\mathrm{R} 4}$ and the singularity $\gamma^{-z}$ for any Newtonian fluid of Prandtl number greater than that of air. The singularities $\gamma^{ \pm y-z}$ are not presented because their critical angles are much smaller than those for $\epsilon^{+z}, \epsilon^{\mathrm{R} 4}$, and $\gamma^{-z}$.

For a given value of $\operatorname{Pr}, \epsilon^{+z}$ is determined by finding the intersection in the $\mathrm{Ra}-\theta$-parameter space of the loci corresponding to the secondary bifurcations $S_{+z}$ and $S_{1} B_{+z}$. Likewise, $\epsilon^{\mathrm{R} 4}$ is determined by finding the intersection of the saddle-node bifurcations $C_{\mathrm{R} 4 \pm}$ and the Hopf bifurcation points involved in the collision. $\gamma^{-z}$ is found by following the bifurcation point $S_{1} B_{-z}$ in the same parameter space until $d \mathrm{Ra}_{c} / d \theta \rightarrow \infty$. The results are shown in Fig. 4 .

The filled area in Fig. 4 represents the range of $\operatorname{Pr}$ and $\theta$ where a single solution exists (characteristic of HSC); i.e., there is no multiplicity of stable solutions (characteristic of RBC). Moreover, from Fig. 4 we can identify three distinctive regions: $\mathbf{A}, \mathbf{B}$, and $\mathbf{C}$, in which the final transition to the single stable solutions occurs for the singularity $\gamma^{-z}(1.47<\operatorname{Pr} \rightarrow$ $\infty)$, the collision $\epsilon^{\mathrm{R} 4}(0.86 \leqslant \operatorname{Pr}<1.47)$, and the collision $\epsilon^{+z}(0.71 \leqslant \operatorname{Pr}<0.86)$, respectively. For any Newtonian fluid with $\operatorname{Pr} \geqslant 0.71$ as a whole, only one stable solution exists if the inclination angle of the cavity is $\theta \gtrsim 25^{\circ}$.

\section{CONCLUSIONS}

In this paper, we have addressed a century-old problem concerning the transition of steady natural convection from Rayleigh-Bénard convection (RBC) to the heated-from-thesides configuration (HSC). Until now, RBC and HSC had been thoroughly studied for the cubical cavity configuration, but the mechanism underlining the RBC-HSC transition was still unknown. As an initial step to understand this transition, we first propose the concept of quasi-Rayleigh-Bénard convection (QRBC) as a real physical approximation to the ideal $\mathrm{RBC}$ and then analyze the effect of a marginal inclination angle on the stability of the system. We show that theoretical results dealing with Rayleigh-Bénard should include a marginal inclination in order to be physically meaningful, especially in the vicinity of instability thresholds. Our analysis then yields comprehensive bifurcation diagrams for QRBC in a cubical cavity filled with air $(\mathrm{Pr}=0.71)$. Furthermore, we demonstrate the existence of several critical angles during the RBC-HSC transition and show that these critical angles are the result of collisions and singularities of bifurcation points that translate in the Ra-parameter space as the inclination angle is increased. Finally, the main critical angles corresponding to two collisions and one singularity are determined for a Newtonian fluid with a Prandtl number larger than air, $0.71 \leqslant \operatorname{Pr} \rightarrow \infty$. These results unveil the transition to a single solution, which is characteristic of HSC, and show that depending on the value of Pr either a collision or a singularity of bifurcation points determines the limit angle for this transition.

\section{ACKNOWLEDGMENT}

We thank Dolors Puigjaner for revising the manuscript and stimulating discussion.
[1] M. Gurnis, Nature 332, 695 (1988).

[2] J. Leconte and G. Chabrier, Nature Geosci. 6, 347 (2013).

[3] D. Braun, N. L. Goddard, and A. Libchaber, Phys. Rev. Lett. 91, 158103 (2003).

[4] M. Krishnan, N. Agrawal, M. A. Burns, and V. M. Ugaz, Anal. Chem. 76, 6254 (2004).

[5] A. D'Orazio, M. Corcione, and G. P. Celata, Int. J. Therm. Sci. 43, 575 (2004).
[6] J. Y. Moon and B. J. Chung, Nucl. Eng. Des. 274, 146 (2014).

[7] M. C. Cross and P. C. Hohenberg, Rev. Mod. Phys. 65, 851 (1993).

[8] A. Baïri, E. Zarco-Pernia, and J. M. García de María, Appl. Therm. Eng. 63, 304 (2014).

[9] A. V. Getling, Rayleigh-Bénard Convection (World Scientific, Singapore, 1998). 
[10] J. Pallares, F. X. Grau, and F. Giralt, Int. J. Heat Mass Tran. 42, 753 (1999).

[11] D. Venturi, X. Wan, and G. E. Karniadakis, J. Fluid Mech. 650, 391 (2010).

[12] D. Puigjaner, J. Herrero, C. Simó, and F. Giralt, J. Fluid Mech. 598, 393 (2008).

[13] H. Bénard, J. Phys. Theor. Appl. 10, 254 (1901).

[14] L. Rayleigh, Phil. Mag. 32, 529 (1916).

[15] J. Pallares, M. P. Arroyo, F. X. Grau, and F. Giralt, Exp. Fluids 31, 208 (2001).

[16] D. Puigjaner, J. Herrero, F. Giralt, and C. Simó, Phys. Fluids 16, 3639 (2004).

[17] G. D. Mallinson and G. Davis, J. Fluid Mech. 83, 1 (1977).

[18] J. E. Hart, J. Fluid Mech. 47, 547 (1971).

[19] K. R. Kirchartz and H. Oertel, Jr., J. Fluid Mech. 192, 249 (1988).

[20] Y.-M. Chen and A. J. Pearlstein, J. Fluid Mech. 198, 513 (1989).

[21] J. Mizushima and T. Adachi, J. Phys. Soc. Jpn. 64, 4670 (1995).

[22] A. Zebib and M. M. Bou-Ali, Phys. Rev. E 79, 056305 (2009).

[23] J. Neustupa and D. Siginer, Nonlinear Anal.-Theor. 123, 68 (2015).

[24] Ş. Yİ̆git, R. J. Poole, and N. Chakraborty, J. Heat Transfer 137, 052504 (2015).

[25] J. F. Torres, D. Henry, A. Komiya, and S. Maruyama, J. Fluid Mech. 756, 650 (2014).
[26] J. F. Torres, D. Henry, A. Komiya, S. Maruyama, and H. Ben Hadid, Phys. Rev. E 88, 043015 (2013).

[27] Y.-P. Hu, Y.-R. Li, and C.-M. Wu, Phys. Fluids 27, 034102 (2015).

[28] A. N. Sharifulin and A. N. Poludnitsin, J. Appl. Mech. Tech. Phys. 55, 462 (2014).

[29] D. Henry and B. Roux, J. Fluid Mech. 195, 175 (1988).

[30] A. Bergeon and E. Knobloch, Phys. Fluids 14, 3233 (2002).

[31] J. F. Torres, A. Komiya, D. Henry, and S. Maruyama, J. Chem. Phys. 139, 074203 (2013).

[32] C. K. Mamun and L. S. Tuckerman, Phys. Fluids 7, 80 (1995).

[33] H. Ben Hadid and D. Henry, J. Fluid Mech. 333, 57 (1997).

[34] D. Henry and H. Ben Hadid, Phys. Rev. E 76, 016314 (2007).

[35] F. Croccolo, F. Scheffold, and A. Vailati, Phys. Rev. Lett. 111, 014502 (2013).

[36] M. Italia, F. Croccolo, F. Scheffold, and A. Vailati, Eur. Phys. J. E 37, 101 (2014).

[37] K. A. Cliffe and K. H. Winters, J. Comput. Phys. 54, 531 (1984).

[38] H. Ozoe, H. Sayama, and S. W. Churchill, Int. J. Heat Mass Tran. 17, 401 (1974).

[39] W. H. Leong, K. G. T. Hollands, and A. P. Brunger, Int. J. Heat Mass Tran. 42, 1979 (1999).

[40] A. Baïri, N. Laraqi, and J. M. García de María, Exp. Therm. Fluid Sci. 31, 309 (2007). 